\title{
The Whipple Collaboration
}

M. Schubnell, ${ }^{1}$ C. W. Akerlof, ${ }^{1}$ M. F. Cawley, ${ }^{2}$ M. Chantell, ${ }^{3}$ D. J. Fegan, ${ }^{4}$ S. Fennell, ${ }^{3}$ K. S. O'Flaherty, ${ }^{4}$ J. A. Gaidos, ${ }^{5}$ J. Hagan, ${ }^{4}$ A. M. Hillas ${ }^{6}$ Y. Jiang, ${ }^{3}$ A. D. Kerrick, ${ }^{7}$ R. C. Lamb, ${ }^{7}$ M. A. Lawrence, ${ }^{3}$ D. A. Lewis, ${ }^{7}$ D. I. Meyer, ${ }^{1}$ G. Mohanty, ${ }^{7}$ M. Punch, ${ }^{3}$ P. T. Reynolds, ${ }^{7}$ A. C. Rovero, ${ }^{3}$ G. Sembroski, ${ }^{5}$ T. C. Weekes, ${ }^{3}$ T. Whitaker, ${ }^{3}$ and C. Wilson ${ }^{5}$

1 University of Michigan, Ann Arbor, MI 48109 USA

2 St. Patrick's College, Maynooth, Co. Kildare, Ireland

${ }^{3}$ Whipple Observatory, Harvard-Smithsonian CfA, Amado, AZ 85645 USA

${ }^{4}$ University College Dublin, Belfield, Dublin 4, Ireland

${ }^{5}$ Purdue University, West Lafayette, IN 47907 USA

${ }^{6}$ University of Leeds, Leeds LS2 9JT, UK

${ }^{7}$ Iowa State University, Ames, IA 50011 USA

\section{ABSTRACT}

We present here the detection of $\mathrm{TeV}$ gamma rays from the extragalactic source Markarian 421. Observations obtained with the Whipple $10 \mathrm{~m}$ imaging Cherenkov telescope in March-June 1992 give a gamma ray signal with statistical significance of $6.3 \sigma$ above background. The excess corresponds to an average flux of 1.5 $10^{-11} \mathrm{~cm}^{-2} \mathrm{~s}^{-1}$ above $0.5 \mathrm{TeV}$, equivalent to 0.3 of that from the Crab Nebula. The estimated location of the source agrees with the position of Mrk 421 to the angular uncertainty of the Whipple instrument ( 6 arc minutes).

\section{INTRODUCTION}

Markarian 421, a giant elliptical galaxy with a nucleus of the BL Lacertae type $e^{[1,2]}$, has been identified as a strong and variable emitter at radio ${ }^{[3-5]}$, optical $^{[2,5]}$, and X-ray ${ }^{[5-7]}$ frequencies. The pattern of variability for Mrk 421 is erratic and variations on time scales of days (optical) and hours (X-ray) are reported ${ }^{[8-10]}$.

The EGRET detector aboard the Compton Gamma Ray Observatory ${ }^{[11]}$ recently extended the observed energy spectrum from Markarian 421 into the $\mathrm{MeV}$. $\mathrm{GeV}$ regime and made it a good candidate for detection at $\mathrm{TeV}$ energies. Although Markarian 421 is the weakest source reported by the EGRET experiment so far $\left(\Phi(E>.1 \mathrm{GeV})=0.14 \times 10^{-6} \mathrm{~cm}^{-2} \mathrm{~s}^{-1}\right)^{[12]}$, it is still close enough $(\mathrm{z}=0.031)^{[13]}$ to avoid possible obscuration at $\mathrm{TeV}$ energies by infrared absorption ${ }^{[14,15]}$.

\section{The Whipple $10 \mathrm{M}$ Cherenkov telescope}

The observations reported in this paper were carried out with the very-highenergy $\gamma$-ray telescope ${ }^{[16]}$ at the Whipple Observatory on Mt. Hopkins in southern 
Arizona $\left(31^{\circ} 41^{\prime}\right.$ north, $110^{\circ} 53^{\prime}$ west, alt $=7685$ feet $)$.

The 10 meter diameter telescope images Cherenkov light from airshowers on a two-dimensional, hexagonal array of 109 fast photomultipliers in the focal plane. The inner 91 one-inch tubes of this high resolution camera have a $0.25^{\circ}$ field of view and are surrounded by an outer ring of 18 two-inch tubes. The total field of view for the camera is $3.5^{\circ}$. A shower image is recorded when at least two out of the inner 91 tubes exceed the threshold of 50 photoelectrons. With this condition, a typical trigger rate of $3 \mathrm{~Hz}$ is obtained.

\section{Shower IMAge Selection}

The vast majority of atmospheric air showers are produced by charged hadronic particles and have to be discriminated against those which are originated by gamma rays. The most sensitive technique yet employed by the Whipple Collaboration for this purpose ('supercuts ${ }^{\prime 17]}$ ) uses four parameters to characterize the approximately elliptical shower image. Two of these are the root-mean-square length and width of the ellipse. A third, 'distance', is the angular distance of the centroid of the shower image from the assumed source location in the image plane. A fourth parameter, 'alpha', gives the orientation of the image. Alpha is defined to be the angle between the major axis of the shower image and a line from its centroid to the assumed source location in the image plane. For $\gamma$-ray showers from a point-like source, alpha should be near $0^{\circ}$ since the elliptical images point to the location of the source in the image plane. The supercuts procedure selects showers with small size, at distances from $0.51^{\circ}$ to $1.1^{\circ}$, and with values of alpha $<$ 15 degrees. Monte Carlo simulations ${ }^{[18,19]}$ and repeated observations of the Crab Nebula ${ }^{[20,21]}$ demonstrate that the Cherenkov light images of air showers induced by $\gamma$-rays can be reliably distinguished from hadronic background.

\section{ANALYSIS AND RESULTS}

Observations of Markarian 421 were made between 24 March and 2 June 1992, for a total of 7.5 hours on-source and an equal amount of time off-source (R.A. $.^{\text {off-source }}=$ R.A. $.^{\text {on-source }}+30^{\prime}$ ). The data presented here have been restricted to observations at elevations greater than $55^{\circ}$.

In Fig. 1a, the alpha distributions for on-source and off-source observations of Mrk 421 are compared after the other supercuts selection criteria have been satisfied. For the region of alpha $<15$ degrees there is a $6.3 \sigma$ excess, with 302 onsource showers and 166 off-source showers. The excess corresponds to an average photon flux of $1.5 \times 10^{-11} \mathrm{~cm}^{-2} \mathrm{~s}^{-1}$ above $0.5 \mathrm{TeV}$, equivalent to 0.3 that of the intensity of the Crab Nebula. If one assumes isotropic emission at a distance of $124 \mathrm{Mpc}$, then the corresponding luminosity is $\sim 10^{43} \mathrm{erg} \mathrm{s}^{-1}$. But as Mrk 421 is known to exhibit jet-like behavior, the actual $\mathrm{TeV}$ luminosity may be considerably less.

For comparison, the alpha distributions for the previously reported observa- 


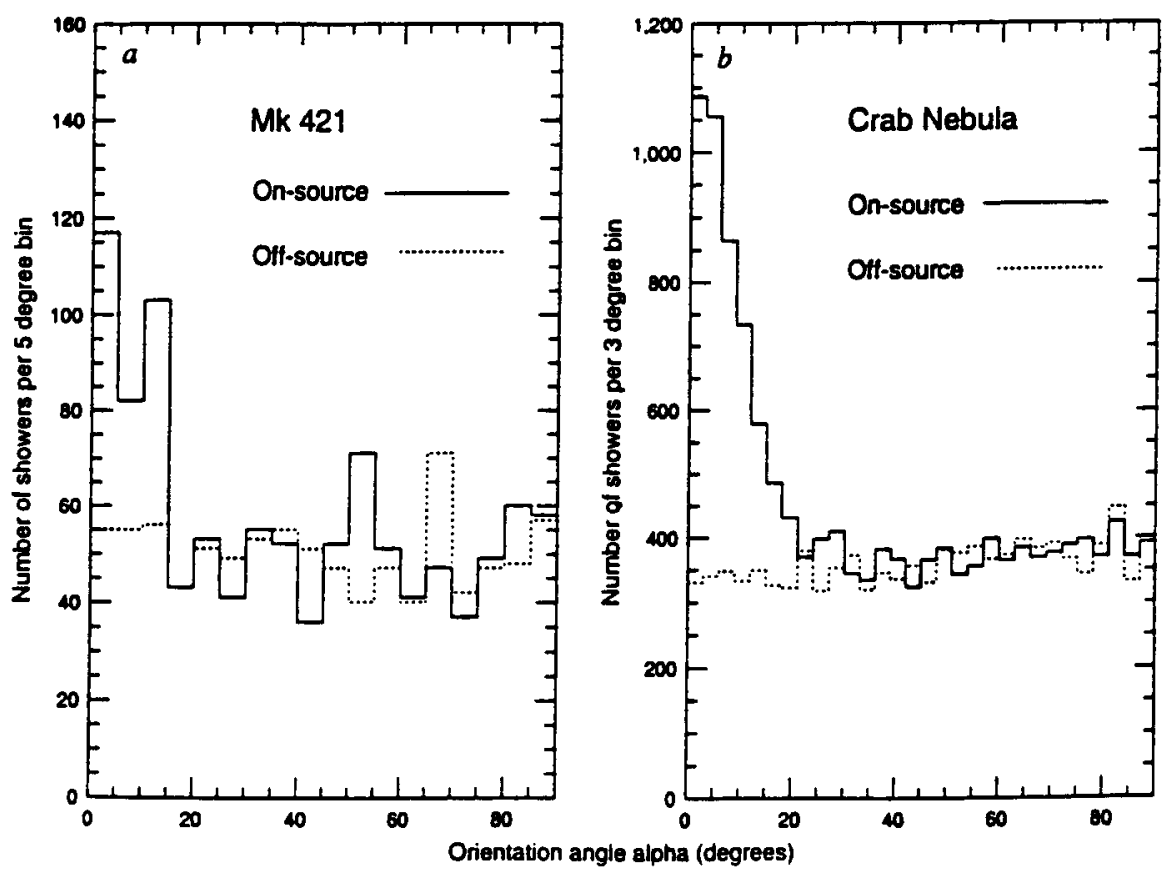

Figure 1. On and off-source orientation angle ('alpha') distributions for $a, M_{k} 421$ and $b$, Crab Nebula ${ }^{[2]}$. The distributions are for those showers for which the other supercuts selection criteria have been satisfied. The supercuts selection value for alpha is $15^{\circ}$.

tions of the Crab Nebula ${ }^{[21]}$ are shown in Fig. 1b. For the Crab Nebula the excess has a statistical significance of $34 \sigma$. The similarity between the Mrk 421 excess in the small-angle region of Fig. 1 with the corresponding excess for the Crab Nebula corroborates the Mrk 421 signal. As a measure of the stability of the Whipple detector, the on-source and off-source datasets contained 77,181 and 76,761 raw, uncut showers, respectively, a difference of only $0.55 \%$. We have investigated the possibility that the excess shown in Fig. 1a may be a systematic effect related to the on- and off-source star fields but find that control observations of other star fields with similar characteristics show null results when they are subjected to the supercuts analysis.

From the observations a two-dimensional map of the source region ${ }^{[23]}$ may be created. Figure 2 shows the map from the observations of Mrk 421. The center of the field of view corresponds to the known direction of the source. The peak seen is within 0.1 degree of this direction.

Mrk 421 is only the second source to be seen by the Cherenkov imaging technique and the first extragalactic source. The power-law energy spectra reported by EGRET $^{[12]}$ for the active galactic nuclei that it has detected are uniformly 

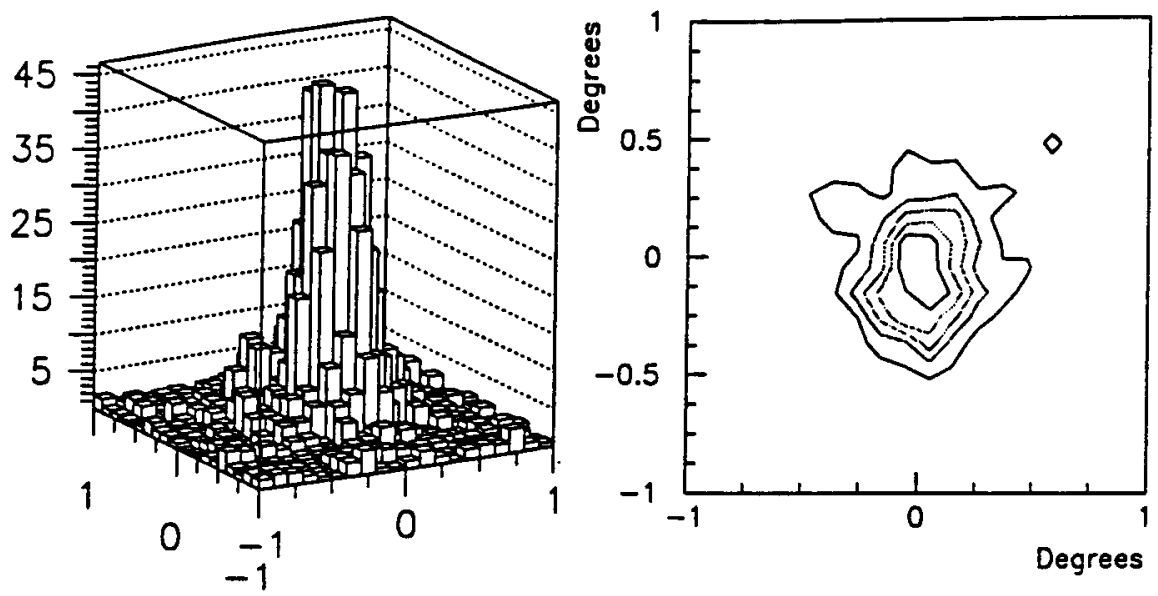

Figure 2. Maps of the on-source observations for Mrk 421 made according to the prescription of ref. [23], figures 4 and 5. The peak intensity lies within $0.1^{\circ}$ of the known location of Mrk 421.

hard, with differential photon spectral indices of two or less. For Mrk 421 the differential power-law index is estimated as $1.9^{[12]}$. The spectral index implied by joining the $100-\mathrm{MeV}$ point with the flux reported here at $0.5 \mathrm{TeV}$ is 2.0 . A first estimate from our data is consistent with a hard energy spectrum at $\mathrm{TeV}$ energies.

\section{ACKNOWLEDGEMENTS}

We thank Kevin Harris and Teresa Lappin for help in obtaining these observations. This research was supported by the US Department of Energy, NASA, the Smithsonian Scholarly Studies Research Fund, and EOLAS, the scientific funding agency of Ireland.

\section{REFERENCES}

[1] Ulrich, M.-H., Kinman, T. D., Lynds, C. R., Rieke, G. H. \& Ekers, R. D. Ap. J. 198 261-266 (1975).

[2] Maza, J., Martin, P. G. \& Angel, J. R. P. Ap. J. 224, 368-374 (1978).

[3] Owen, F. N., Porcas, R. W., Mufson, S. L. \& Moffett, T. J. Astron. J. 83, 685-696 (1978).

[4] Zhang, F. J. \& Bååth, L. B. Astro. Ap. 236, 47-52 (1990).

[5] Mufson, S. L., Hutter, D. J., Kondo, Y., Urry, C. M. \& Wisniewski, W. Z. Ap. J. 354, 116-123 (1990). 
[6] Mushotzky, R. F., Boldt, E. A., Holt, S. S. \& Serlemitsos, P. J. Ap. J. (Letters) 232, L17-L19 (1979).

[7] George, I. M., Warwick, R. S. \& Bromage, G. E. M. N. R. A. S. 232, 793-808 (1988).

[8] Miller, H. R., Ap. J. (Letters) 201, L109 (1975).

[9] Ricketts, M. J., Cooke, B. A., and Pounds, K. A. Nature 259, 546 (1976).

[10] Purton, C. R., Kojoian, G., and Dickinson, D. F. Pub. A. S. P. 89, 119 (1977).

[11] Gehrels , N. \& Kniffen, D. A. in Proc. 22nd International Cosmic Ray Conf. (Dublin) 5, 48-58 (1991).

[12] Fichtel, C. E. et al. to be published in Proc. Compton Observatory Symposium (St. Louis) (1992).

[13] Burbidge, G \& Hewitt, A. Astron. J. 92, 1 (1987).

[14] Nikishov, A. J. Soviet Physics - JETP 14, 393-394 (1962).

[15] Stecker, F. W., De Jager, O. C., \& Salamon, M. H. Ap. J. (Letters) 390, L49-L52 (1992).

[16] Cawley, M. F. et al. Exper. Astr. 1, 173-193 (1990).

[17] Punch, M. et al. in Proc. 22nd International Cosmic Ray Conf. (Dublin) 1, 464-467 (1991).

[18] Hillas, A. M. in Proc. 19th International Cosmic Ray Conf. (La Jolla) 3, 445-448 (1985).

[19] Macomb, D. J. \& Lamb, R. C. in Proc. 21st International Cosmic Ray Conf. (Adelaide) 2, 435-438 (1990).

[20] Weekes, T. C. et al. Ap. J. 342, 379-395 (1989).

[21] Vacanti, G. et al. Ap. J. 377, 467-479 (1991).

[22] Punch, M. et al. Nature 358, 477 (1992).

[23] Akerlof, C. W. et al. Ap. J. (Letters) 377, L97-L100 (1991). 